(C) 2016 IEEE. Personal use of this material is permitted. Permission from IEEE must be obtained for all other uses, in any current or future media, including reprinting/republishing this material for advertising or promotional purposes, creating new collective works, for resale or redistribution to servers or lists, or reuse of any copyrighted component of this work in other works. 


\title{
The Effect of Absorption Losses on the Optical Behaviour of Macroporous Silicon Photonic Crystal Selective Filters
}

\author{
D. Vega, D. Cardador, T. Trifonov, M. Garín, Á. Rodríguez, Senior IEEE
}

\begin{abstract}
The effect of losses in photonic crystal structures on transmittance and reflectance has been studied by numerical techniques, and compared to fabricated macroporous silicon samples. Material absorption and tolerances of the pores play an essential role determining performance in optical applications. The relevance of the structure geometry has been investigated through the porosity. The considered photonic crystals in this work are 3-d macroporous silicon structures fabricated by electrochemical etching with pore profile modulation, and defect inclusion. The examined structures are intended to work with quasi-normal light incidence as selective filters. The effect of losses on the forbidden band and resonance is studied and reported for both transmittance and reflectance. The reflectance peak is less affected by losses, while transmitted light experiences a noticeable reduction even for small absorption factors. This has critical implications for the performance of these structures as transmission filters. Furthermore, it is shown that an optimum can be found for a given absorption coefficient by choosing the appropriate porosity.
\end{abstract}

Index Terms - Photonic crystals Macroporous Silicon, Silicon photonics, Electrochemical etching, Optical losses

\section{INTRODUCTION}

$\mathrm{P}$ HOTONIC crystals (PC) are becoming increasingly attractive for both research and market applications. PCs are artificially structured materials that show unique optical characteristics. That makes them of high interest in a broad range of application areas, such as optical communications or sensing [1]-[3]. PCs help in miniaturization and cost reduction, and have been proposed for several applications like high-selectivity tuned filters [4], optical resonators [5], waveguides [6], selective emitters [7], [8], and thermophotovoltaics [9]. PCs can be implemented with several materials. Porous silicon (PS) is the base of PC structures made of silicon. In particular macroporous silicon $(\mathrm{MpS})$, a specific type of PS which has pores with diameter larger than $50 \mathrm{~nm}$, and with a regular structure, has been suggested for many of the aforementioned applications [8], [10], [11]; the study of this material is the main concern in this paper.

Photonic bandgaps (PBG) of PCs can be easily engineered to suit one's needs. Furthermore, high quality resonant modes can be achieved by embedding cavities in a PC lattice. These cavities can be produced by disturbing the periodicity of a regular PC structure [12]. Several factors influence the performance of the defect state, like PBG size and defect position. Research about PC optical behaviour can be found in literature [13], [14].

For optical applications, losses in the PC structure can be of great importance. A significant number of works can be found dealing with losses for photonic crystal waveguides. Most PC waveguides in these works are based on two-dimensional (2D) structures which use in-plane light propagation [15], [16]. On the other hand, there is less published research on the effect of losses for out-of-plane wave propagation [17], [18].

Macroporous silicon has some distinctive advantages over other PS implementations. It can be fabricated using electrochemical etching (EE) in dilute hydrofluoric acid (HF). This technique allows to precisely position the pores in a desired arrangement [19], and pores grow as smooth cylindrical surfaces [20]. It also makes possible profile modulation. Though EE has limitations in achievable minimum size, and etching time, it has advantages such as ease of fabrication, and surface quality [21].

Regarding the study of losses in macroporous silicon, work is focused to particular cases. In general, they are about PC waveguides, using in-plane propagation, where losses were mainly attributed to: scattering due to surface roughness [22], impedance mismatch [23], and leakage [24]. An interesting result from the study of PC waveguides, was the development of disorder modes theory [25]. One of the pioneering works that dealt with 3D PCs and examined losses for out of plane propagation in a systematic way [14], was limited by technology, to microwave PCs made of alumina rods. In this work, a

This paper was submitted on July 17, 2015. This work was supported in part by the Spanish Commission of Science and Technology under Grant TEC201348147-C6-2-R.

D.V., D.C., M.G., and A.R., are with the Electronic Engineering Department (EEL), Universitat Politècnica de Catalunya (UPC), Barcelona 08034, Spain (email: didac.vega@upc.edu; david.cardador@upc.edu; mgarin@eel.upc.edu; angel.rodriguez@upc.edu).

T.T. is with the Centre de Recerca en Nano-Enginyeria (CrNE), Universitat Politècnica de Catalunya (UPC), Barcelona 08028, Spain (e-mail: trifon.todorov@upc.edu). 
general analysis of losses is done, and their effect on selective filters in the mid-IR range is investigated for out-of-plane propagation. The results are then compared to MpS structures fabricated and measured in our facilities.

Other works have studied the influence of impurities in the silicon bulk. The Franz-Keldysh effect [26] and the Wannier-Stark effect [27] were reported. Though their effect should be negligible, a notable point risen from these works is the possibility of trapped charge effects in PCs coatings. Also the surface finish plays an important role. Some works involving microporous silicon [28] found that surface resistivity increased for low doped samples as revealed by IR measures. EE creates a thin layer of microporous silicon, but this layer is very thin and common post-treatment of EE silicon include surface passivation. Also, in [29] surface resonant modes are analysed in the air-PC interface. Though the reported effect is small, some kind of impedance matching in the interface is recommended.

Different factors have been identified as causes of absorption in PCs. For instance, we can consider intrinsic material losses due to the pure silicon matrix, extrinsic losses due to impurities in the form of free electrons, and scattering losses due to shape irregularities. One of the more appealing applications of PCs is their use as gas sensing devices [6], [8], [11]. Making use of resonating structures, it is possible to construct devices with sharp resonances tuned to an absorption line of the target substance [30]. These PCs can be either used as filters, or as selective emitters. PCs have the distinct advantage when compared to traditional metal-oxide (MOX) or polymer sensors [31], [32]. Selectivity and response time are two of the most notable [33].

To achieve the anticipated selectivity, wide PBG and sharp resonant peaks are needed, and losses in the PC can have a detrimental effect on the optical performance: dynamic range of PBGs reduces, and resonant peaks have lower quality-factors. This will negatively impact selectivity and sensitivity. Separating the various loss mechanisms during characterization of samples can be very complicated. It is preferable to define a single factor for a practical approach for measuring the optical behaviour in the considered wavelength range. In this work, the aim is to study how losses, whatever the involved mechanisms, affect the reflectance and transmittance spectra of the MpS samples fabricated, for their use in gas sensing applications. One of the goals of this work is to fill the gap in the study of out-of-plane light propagation, and for MpS in particular. Normal and quasi-normal incidence angle (parallel to the pore axis) is investigated by numerical simulation and compared to measured samples in the mid-IR range with a Fourier Transform Infrared Spectroscopy (FT-IR) spectrometer. FDTD is used for the numerical study, with an approximated shape to the real etched MpS samples fabricated in our facilities.

\section{SAMPLE PREPARATION AND FABRICATION}

Samples of macroporous silicon PCs were fabricated by the EE technique. In particular, samples of $4 \mu \mathrm{m}$ pitch were made. The samples were defined with a regular square lattice for the pore growth sites and the radius was modulated obtaining a sinelike profile in depth [11], see Fig. 1(a). A defect was introduced halfway the total pore depth by suppressing one of the modulations, and leaving a constant diameter section [34], see Fig. 1(b). Depth periodicity was set to be about the lattice pitch. N type silicon wafers with $3-5 \Omega \cdot \mathrm{cm}$ resistivity and $\langle 100\rangle$ crystallinity were used. The EE technique allows control of the pore diameter by independent regulation of cell potential and current. A complete description of the process can be found elsewhere [19], [35]. After EE, a membrane is created by etching the backside remaining bulk silicon [34].

For the $4 \mu \mathrm{m}$ pitch sample, the modulated depth is about 45 micrometres. At the opening of the pore an initial taper remains from the inverted pyramids created in the initial pattern definition step. The EE method [19], allows creating 3-d PCs by changing the pore radius as the pore tip advances. This is a slow process, resulting in smooth profiles for the pores. The gentle transition at the MpS surface helps to reduce the reflection at the interface, which is desirable for light coupling when coming from outside the photonic crystal, but the opposite is sought at the defect site: a better confinement is achieved with an abrupt change in the interface.

As can be seen from the SEM characterization of the fabricated samples, depicted in Fig. 1, the resulting as-etched surfaces for the porous structures are smooth and of high quality: i.e. the surfaces have little microporous silicon and the shape is regular and stable across the whole sample. Therefore, the EE macroporous silicon is suitable for photonic applications. The thin oxidized microporous layer created during EE has not noticeable optical impact, and is typically removed after etching. Unless other treatments are performed, native oxide will be present, but it is also optically unimportant. The fabricated samples with and without defect are shown in Fig. 3 for comparison.

The remaining PC above and below of the defect act as distributed Bragg reflectors (DBR), hence the defect can be equated to a Fabry-Perot (FP) cavity, as is depicted schematically in Fig. 2. The fabricated FP cavity results in a resonance mode that can be tuned at design time to a selected gas absorption line. The relationship between the defect length and diameter is crucial for the resonant state placement. Also, the overall PC porosity is of great importance for its placement. Porosity is defined as the volume fraction of silicon over the total unitary cell volume, as the ratio: $p=V_{S i} / V_{t o t}$. For the fabricated samples, porosity is about $30.4 \%$.

\section{OPTICAL MEASUREMENTS}

The fabricated samples where measured using a Bruker Optics' Vertex FT-IR spectrometer in the mid-IR range, from $5 \mu \mathrm{m}$ to $28 \mu \mathrm{m}$ approximately. Samples were measured both in specular reflection and transmission. Specular reflection measurements 
were performed with an aperture of $1 \mathrm{~mm}$ and a resolution of $8 \mathrm{~cm}^{-1}$ at quasi-normal incidence $\left(13^{\circ}\right)$. All measurements have been normalized to the source spectrum, directly for the transmittance and using a gold mirror for the reflectance spectra. The lattice was aligned to the $\Gamma-M$ direction (along one lattice axis in the surface plane).

In Fig. 4 is shown the measured reflectance and transmittance spectra for the $4 \mu \mathrm{m}$ samples with a defect. The PBG is clearly identified in the range from $\lambda=15 \mu \mathrm{m}$ to $23 \mu \mathrm{m}$, and the resonance peak is located at $f_{c} 20 \mu \mathrm{m}$ approximately.

\section{NUMERICAL STUDY}

The studied PC structures have been numerically analysed before fabrication to extract the dispersion diagram, and to tune the defect. For the initial simulations the plane wave expansion method (PWM) has been used employing the MPB software package [36]. All simulations have been performed in three dimensions (3D). To obtain the band structure for the ideal PC, a single unitary cell has been considered, see Fig. 2. As shown in Fig. 1 and Fig. 3, the pore shape can be approximated by a composition of ellipsoids and cylinders. This model of the structure provides a good enough match to the physical one. All dimensions have been normalized to the pore pitch, as the fabricated structures are simple scaled replicas of the same profile. Therefore, the obtained results are given in normalized units $\ell$, which are transported to the correct physical frequency range by a scaling operation. The unitary cell size is a $1 \ell \times 1 \ell \times 1 \ell$ box. The major axis of the ellipsoids was aligned to the pore (Z) axis and kept at a constant ratio of 1.015 to the minor axis (the $X$ and $Y$ axis), resulting in a prolate ellipsoid; the cylinders were likewise oriented along the $Z$ axis. The porosity is controlled by changing the maximum diameter ( $D_{\max }$ in Fig. 2 ) from $0.4 \ell$ to $1 \ell$, and keeping the minimum diameter ( $D_{\min }$, that of the cylinders) constant to $0.4 \ell$.

The band structure obtained for the square lattice of pores, demonstrates the existence of a PBG between the first band and the second band for normal light incidence ( $\Gamma-$ A direction). The solid line in Fig. 5 shows that the PWM bandgap evolves with the porosity from non-existent in the $p=12 \%$ case (only straight pores) to a $29.8 \%$ gap for $p=53 \%$ (touching ellipsoids between pores). Afterwards, the defect was inserted in the PC. This was simulated with a superlattice where one of the modulations in the $Z$ direction was suppressed, keeping it at constant diameter, and made slightly longer. As commented, the defect behaves as a resonant cavity with a state in $f_{c}$. This is shown in the field plots in Fig. 6 , where the power density achieves its maximum at the defect site. To avoid cavity leakage and achieve a better quality factor, the resonant state must be as far away from the band edges as possible. From Fig. 5, it can be seen that $f_{c}$ changes with porosity. The PWM method predicts that for $p<24 \%$, the defect should disappear. From these results, to improve the Q-factor, one should increase $p$. Nevertheless, a limit is reached when adjacent pores touch each other.

To study the effect of losses in a finite PC structure, the Optiwave's OptiFDTD software suite was used, which uses the finitedifference time-domain method (FDTD). The initial simulations focused on the lossless case of the structures in Fig. 3(b). The end of the pore was continued with a region of bulk silicon with a straight pore. Periodic boundary conditions were set on the $X Z$ and $Y Z$ walls while perfect absorbing layers (PML) were used for the top and bottom of the simulation cell. The illumination was done with a plane wave coming from the top and impinging on the front face of the PC. The light wave was a Gaussian pulse with a linewidth of $\Delta \bar{\lambda}=4 \ell$ and a central wavelength about $\bar{\lambda}=5 \ell$. The reflection and transmission spectra were obtained by placing plane detectors $1 \ell$ before the front face of the PC, and $5 \ell$ after the pore end, respectively. The spectra were calculated by integrating the power flux through the detector planes and normalizing to the incident wave. The effect of porosity was also investigated by varying the maximum diameter of the modulation, $D_{\max }$. Afterwards, absorption was added by setting a non-null value for the imaginary part of the dielectric constant of silicon. For these simulations, a constant imaginary part was used, which results in a constant spectral attenuation. As was found for free carrier absorption [37], the reported losses at the wavelengths of interest in this work, for low doped silicon, are very small, and therefore neglected. As other loss mechanisms dominate at room temperature, the simple model used here is adequate. Additionally, narrowband features, like the surface modes described by [29], are of little importance. On the contrary, light scattering by surface roughness or overall geometrical irregularities, contributes a far larger amount to losses, in an almost frequency-independent way. The effect of absorption was studied by varying the extinction factor $\kappa$ in a range from lossless $\left(\kappa=0 \rightarrow \epsilon^{\prime \prime}=0\right)$ to lossy $\left(\kappa=0.04 \rightarrow \epsilon^{\prime \prime} \approx 0.23\right)$, and the results shown in Fig. 7. To perform these simulations, the refraction index for the silicon bulk material was set to $n \approx 3.47$, which is a suitable reference value for the mid-IR region.

\section{A. On Porosity \& Q-factor}

As has been said, the inclusion of a defect in the periodic PC structures creates a resonance. This can be clearly seen as the transmittance peak, and the reflectance dip in the middle of the PBG in Fig. 4 and Fig. 7. For the generated peak it is possible to use the standard definition of quality factor (Q-factor) as the ratio of the peak amplitude to its full with half maximum (FWHM): $Q=f_{c} /$ FWHM. As was shown in Fig. 5, there exists a dependency of the peak frequency with respect to porosity, thus a change in the diameter of the pore, will affect the peak position and amplitude. This was studied to find an optimum, and determine how it related to losses. To find out the latter case, the peak amplitude and Q-factor were considered for each porosity value.

\section{RESULTS \& DISCUSSION}

In this paper different simulation methods are used to evaluate and study the MpS structures later fabricated and measured. 
The different simulation methods have a reasonable match between them. Indeed, in Fig. 5 it can be seen that the PBG edges of both the PWM and FDTD data closely fit. For the resonance peak central frequency, there is also good agreement. An interesting difference between both methods is the existence of a peak for the FDTD results at lower porosities than predicted by PWM; this shown by the symbols in Fig. 5. This may be due to the finite structure used in the FDTD case.

In Fig. 8, the spectral region corresponding to the bandgap is shown for both the measured sample and the lossless FDTD case. It can be seen that reasonable coincidence is achieved. Almost $100 \%$ reflection in the PBG is seen on both the real and simulated spectra, but in the simulation results, a deeper peak is obtained than for the measured PC. In contrast, for the practically null transmitted signal in the simulated PBG, a small signal can be detected for the measured sample while the peak level is practically matched in simulations and in measurements. This small drop in the reflection peak can have several causes. It can be argued that material absorption due to free carriers in doped silicon is to be considered. Nevertheless, as the literature suggests [37], [38], for the actual doping levels used in the fabricated samples (about $1.5 \cdot 10^{15} \mathrm{~cm}^{-3}$ for the $4 \mu \mathrm{m}$ pitch Mps sample), the absorption coefficient should be $\alpha \ll 50 \cdot \mathrm{cm}^{-1}$ in the working wavelength range, and mostly caused by lattice phonon scattering. There is also the small angle of incidence, and the fact that it is a specular reflection measure. Also for the measured spectra, signal windowing and instrument resolution may have to be considered. Considering all these points, the combined effects of the air-PC interface, shape variance and measurement set-up, should be able to explain the observed differences.

Overall, good agreement is achieved for both transmitted and reflected spectra with the lossless simulation. Adding a minute amount of losses to the material model, for instance a small extinction factor $\kappa$ about 0.01 , rapidly breaks the concordance between the simulated and the measured spectra, especially for the transmitted part. In the simulations, any amount of losses decreases the spectra. Comparing the behaviour of the transmittance, it can be seen that the PBG has noticeable signal level, which contradicts the simulation data. The conclusion from this, is that, indeed, material losses are negligible (at least for the doping levels of the samples in this work), and that the observed behaviour must be due to scattering induced by disorder in the $\mathrm{PC}$, as suggested above.

In Fig. 7 the evolution of the spectra is shown for increasing absorption coefficient $\kappa$. It is found that transmittance decreased more rapidly than reflectance. This was further investigated, and the peak variation with respect to $\kappa$ plotted in Fig. 9. This shows that the transmitted intensity has the expected exponential decrease, but also does the reflectance: $I=I_{0} e^{l^{\prime} \kappa}$, where $I$ is the wave intensity, and $I_{0}$ is the reference intensity (lossless case, $\left.\kappa=0\right), \kappa$ is the extinction coefficient in Nepers and $l$ ' is a unitless coefficient loosely related to the effective distance the wave travels in the PC. Looking at Fig. 9, the reflected peak slope is gentle: $l^{\prime} \approx-19$, while for the transmitted wave, the fitted $l^{\prime}$ factor is much greater: $l^{\prime} \approx-49$, i.e. two and a half times stronger loss. As said, this is expected for the transmitted light: the well-known case for a plane wave travelling through a lossy medium, but the observed behaviour of reflectance is striking. Bulk intrinsic Si has a 30\% reflectance at IR wavelengths, and a small absorption should not change it significantly. In the MpS PC, the reflected light has to travel about 5 to 7 periods for near total reflection. Thus the optical path length for the reflected light should be around 10 to 12 periods. This distance is similar to that of the transmitted wave for the presented PC, and a similar absorption should be expected. Contrary to this, it seems that the effective distance the reflected wave has to travel is just about 3 periods. This can be explained looking at Fig. 6(a): it can be seen that the $E$ field is stronger in the air part of the defect site, and as Si losses increase, less energy will penetrate the dielectric. Inside the cavity, as Fig. 6(a) shows, energy is concentrated in the Si, but as losses increase, energy will shift to the air portion and be less attenuated. Furthermore, few periods are needed to obtain good reflectivity, so the wave at the resonant frequency $f_{c}$ will see little absorption to reach the cavity.

Regarding the dependence of the peak with porosity, in Fig. 10 is found that an optimum Q-factor is given for a porosity $p \approx$ $35 \%$, though the peak maximum is at a lower porosity $p \approx 26 \%$. This follows the theoretical PWM results of Fig. 5. The initial simulations suggested that better results would be obtained for porosities around $40 \%$, while remaining fairly constant for greater $p$. This is further studied in Fig. 11, where the interesting characteristic is the steeper slope of the higher porosity PC.

\section{CONCLUSION}

In this work, the effect of losses has been studied for a MpS PC with a defect cavity. The reflection and transmission spectra in the mid-IR range have been analysed numerically for light propagation in a normal or quasi-normal incidence angle, along the pores' axis, in the presence of absorption losses in the Si substrate. Samples have been fabricated with a square lattice arrangement of the pores and a $4 \mu \mathrm{m}$ pitch. The samples have been measured and compared with the numerical results; achieving an overall good match between the measured data and the simulated values. The considered PCs have a plane defect embedded in the structure that gives rise to a resonance peak at wavelengths inside the PCs' optical bandgap. It has been observed that good agreement between simulations and measurements is attained for the lossless case. The results of this work show that material losses can be neglected, and that the main loss mechanism is scattering due to surface roughness and structure disorder. Adding a small amount of absorption in the simulations can help adjusting the theoretical results and the measurements, but usually considering lossless materials for the simulations, will result in acceptable results. Both reflectance and transmittance reduce as the extinction factor increases; this has been the case, but, apparently, the reflectance is less sensitive than transmittance. Also, the theoretical transmitted spectra seem to differ from the measured data at the PBG, as we can appreciate a small signal level for the 
measures that is not present in the results of simulation. This discrepancy cannot be solved by adding absorption to the material, and it can only be explained because of the existence of geometrical variance. Furthermore, it has been established that at $f_{c}$, the electric field is mostly concentrated in the air part of the defect, thus reducing the impact of material losses.

The dependence of the resonance peak has been analysed with respect to the porosity. We have found that the defect $\mathrm{Q}$ values depend on the PC porosity, and that there is an optimum porosity that maximizes the attainable Q-factor. Interestingly, this optimum does not coincide with the largest resonance peak, which happens at a lower porosity value. This introduces a tradeoff between filter selectivity and signal level.

To sum up, the results here presented show that for a MpS PC selective filter, losses originated by material absorption can often be neglected for the mid-IR range. This implies that the reasons of the drop off of optical performance of fabricated photonic crystals of this kind are mainly due to extrinsic factors such as surface roughness.

\section{ACKNOWLEDGMENT}

The authors would like to thank J. Calderer, R. Alcubilla and P. Eglitis for helpful discussions and assistance during the preparation of this work.

\section{REFERENCES}

[1] G. Roelkens, S. Stankovic, S. Keyvaninia, D. Vermeulen, M. Muneeb, R. Baets, and D. Van Thourhout, "III-V/silicon photonic integrated circuits for communication applications," 16th Opto-Electronics Commun. Conf., pp. 840-841, 2011.

[2] H. Alipour-Banaei and F. Mehdizadeh, "Significant role of photonic crystal resonant cavities in WDM and DWDM communication tunable filters," Optik (Stuttg)., vol. 124, no. 17, pp. 2639-2644, 2013.

[3] I. Suárez, V. Chirvony, D. Hill, and J. Martínez-Pastor, "Simulation of surface-modified porous silicon photonic crystals for biosensing applications," Photonics Nanostructures - Fundam. Appl., vol. 10, no. 3, pp. 304-311, 2012.

[4] P. B. Deotare, L. C. Kogos, I. Bulu, and M. Loncar, "Photonic crystal nanobeam cavities for tunable filter and router applications," IEEE J. Sel. Top. Quantum Electron., vol. 19, no. 2, p. 3600210, 2013.

[5] X. Serey, S. Mandal, and D. Erickson, "Comparison of silicon photonic crystal resonator designs for optical trapping of nanomaterials.," Nanotechnology, vol. 21, no. 30, p. 305202, 2010.

[6] C. Charlton, B. Temelkuran, G. Dellemann, and B. Mizaikoff, "Midinfrared sensors meet nanotechnology: Trace gas sensing with quantum cascade lasers inside photonic band-gap hollow waveguides," Appl. Phys. Lett., vol. 86, pp. 1-3, 2005.

[7] S. Cheylan, T. Trifonov, A. Rodriguez, L. F. Marsal, J. Pallares, R. Alcubilla, and G. Badenes, "Visible light emission from macroporous Si," Opt. Mater. (Amst)., vol. 29, no. 2-3, pp. 262-267, Nov. 2006.

[8] R. B. Wehrspohn, S. L. Schweizer, B. Gesemann, D. Pergande, T. M. Geppert, S. Moretton, and A. Lambrecht, "Macroporous silicon and its application in sensing," Comptes Rendus Chim., vol. 16, pp. 51-58, 2013.

[9] K. a Arpin, M. D. Losego, A. N. Cloud, H. Ning, J. Mallek, N. P. Sergeant, L. Zhu, Z. Yu, B. Kalanyan, G. N. Parsons, G. S. Girolami, J. R. Abelson, S. Fan, and P. V Braun, "Three-dimensional self-assembled photonic crystals with high temperature stability for thermal emission modification.," Nat. Commun., vol. 4, p. 2630, 2013.

[10] H. Föll, M. Christophersen, J. Carstensen, and G. Hasse, "Formation and application of porous silicon," Mater. Sci. Eng. R Reports, vol. 39, no. 4, pp. 93141, Nov. 2002.

[11] R. B. Wehrspohn, S. L. Schweizer, J. Schilling, T. Geppert, C. Jamois, R. Glatthaar, P. Hahn, A. Feisst, and A. Lambrecht, “Application of Photonic Crystals for Gas Detection and Sensing," in Photonic Crystals, Advances in design, fabrication and characterization, 2006, pp. $238-246$.

[12] S. A. Rinne, F. García-Santamaría, and P. V. Braun, "Embedded cavities and waveguides in three-dimensional silicon photonic crystals," Nature Photonics, vol. 2, no. 1. pp. 52-56, 2008.

[13] G. Mertens, R. B. Wehrspohn, H.-S. Kitzerow, S. Matthias, C. Jamois, and U. Gösele, "Tunable defect mode in a three-dimensional photonic crystal," Appl. Phys. Lett., vol. 87, no. 24, p. 241108, 2005.

[14] M. Sigalas, K. Ho, R. Biswas, and C. Soukoulis, "Theoretical investigation of defects in photonic crystals in the presence of dielectric losses," Physical Review B, vol. 57, no. 7. pp. 3815-3820, 1998.

[15] A. Di Falco, M. Massari, M. G. Scullion, S. A. Schulz, F. Romanato, and T. F. Krauss, "Propagation losses of slotted photonic crystal waveguides," IEEE Photonics J., vol. 4, no. 5, pp. 1536-1541, 2012.

[16] M. J. Cryan, "On the modeling of losses in short length photonic crystal waveguides," J. Light. Technol., vol. 27, no. 21, pp. 4841-4847, 2009.

[17] R. Iliew, C. Etrich, T. Pertsch, and F. Lederer, "Minimization of out-of-plane losses of photonic crystal membranes," Phys. Rev. B - Condens. Matter Mater. Phys., vol. 80, no. 3, 2009.

[18] M. Sigalas, R. Biswas, K. Ho, and C. Soukoulis, "Theoretical investigation of off-plane propagation of electromagnetic waves in two-dimensional photonic crystals," Physical Review B, vol. 58, no. 11. pp. 6791-6794, 1998.

[19] V. Lehmann, "The Physics of Macropore Formation in Low Doped n-Type Silicon," Journal of The Electrochemical Society, vol. 140. p. $2836,1993$.

[20] T. Trifonov, A. Rodríguez, L. F. Marsal, J. Pallarès, and R. Alcubilla, "Macroporous silicon: A versatile material for 3D structure fabrication," Sensors Actuators A Phys., vol. 141, no. 2, pp. 662-669, Feb. 2008.

[21] S. Matthias, F. Müller, J. Schilling, and U. Gösele, "Pushing the limits of macroporous silicon etching," Appl. Phys. A, vol. 80, no. 7, pp. 1391-1396, Apr. 2005.

[22] N. Mann, S. Combrié, P. Colman, M. Patterson, A. De Rossi, and S. Hughes, "Reducing disorder-induced losses for slow light photonic crystal waveguides through Bloch mode engineering.," Opt. Lett., vol. 38, no. 20, pp. 4244-7, 2013.

[23] Q. V. Tran, S. Combrí, P. Colman, and A. De Rossi, "Photonic crystal membrane waveguides with low insertion losses," Appl. Phys. Lett., vol. 95, no. 6, 2009.

[24] Y. Kawaguchi, K. Saitoh, and M. Koshiba, "Analysis of leakage losses in one-dimensional photonic crystal coupled resonator optical waveguide using 3-D finite element method," J. Light. Technol., vol. 28, no. 20, pp. 2977-2983, 2010.

[25] V. Savona, "Electromagnetic modes of a disordered photonic crystal," Phys. Rev. B, vol. 83, no. 8, p. 085301, Feb. 2011.

[26] L. a. Karachevtseva, V. I. Ivanov, O. O. Lytvynenko, K. a. Parshin, and O. J. Stronska, "The impurity Franz-Keldysh effect in 2D photonic macroporous silicon structures," Appl. Surf. Sci., vol. 255, no. 5 PART 2, pp. 3328-3331, 2008.

[27] L. Karachevtseva, S. Kuchmii, O. Lytvynenko, F. Sizov, O. Stronska, and A. Stroyuk, "Oscillations of light absorption in 2D macroporous silicon structures with surface nanocoatings," Appl. Surf. Sci., vol. 257, no. 8, pp. 3331-3335, 2011. 
[28] S. Hilbrich, W. Thei $\beta$, R. Arens-Fischer, O. Glück, and M. . Berger, "The influence of the doping level on the optical properties of porous silicon," Thin Solid Films, vol. 276, no. 1-2. pp. 231-234, Apr-1996.

[29] G. V. Li, E. V. Astrova, S. A. Dyakov, A. Baldycheva, T. S. Perova, S. G. Tikhodeev, and N. A. Gippius, "Surface Tamm states in a photonic crystal slab with asymmetric termination," Phys. Status Solidi - Rapid Res. Lett., vol. 7, no. 7, pp. 481-484, 2013.

[30] D. Vega, F. Marti, A. Rodriguez, and T. Trifonov, "Macroporous silicon for spectroscopic $\mathrm{CO}_{2}$ detection," in IEEE SENSORS 2014 Proceedings, 2014 , pp. 1061-1064.

[31] E. Llobet, "Gas sensors using carbon nanomaterials: A review," Sensors and Actuators, B: Chemical, vol. 179. pp. $32-45,2013$.

[32] K. Arshak, E. Moore, G. M. Lyons, J. Harris, and S. Clifford, "A review of gas sensors employed in electronic nose applications," Sensor Review, vol. 24. pp. 181-198, 2004.

[33] R. V. Nair and R. Vijaya, "Photonic crystal sensors: An overview," Progress in Quantum Electronics, vol. 34. pp. 89-134, 2010.

[34] T. Trifonov, A. Rodríguez, L. F. Marsal, J. Pallarès, and R. Alcubilla, "Two- And three-dimensional microstructures produced by electrochemical etching of silicon," in 2005 Spanish Conference on Electron Devices, Proceedings, 2005, vol. 2005, pp. 233-236.

[35] S. Matthias, F. Muller, R. Hillebrand, J. Schilling, and U. Gosele, "Three-dimensional silicon-based photonic crystals fabricated by electrochemical etching," First IEEE Int. Conf. Gr. IV Photonics, 2004., 2004.

[36] S. Johnson and J. Joannopoulos, "Block-iterative frequency-domain methods for Maxwell's equations in a planewave basis," Opt. Express, vol. 8, no. 3, p. 173, Jan. 2001.

[37] W. Spitzer and H. Y. Fan, "Infrared Absorption in n -Type Silicon," Phys. Rev., vol. 108, no. 2, pp. 268-271, Oct. 1957.

[38] K. Winer and M. Cardona, "Theory of infrared absorption in silicon," Phys. Rev. B, vol. 35, no. 15, pp. 8189-8195, May 1987.

D. Vega received the B.S. degree in telecommunications engineering from the Universitat Politècnica de Catalunya, Barcelona in 2009. He is currently pursuing his $\mathrm{PhD}$ degree in electronic engineering. His research interests include photonics, nanomaterials and macroporous silicon.

D. Cardador received the B.S degree in physics from the Universitat de Barcelona, Barcelona in 2008, and the M.S. degree in electronic engineering from the Universitat Politècnica de Catalunya, Barcelona in 2013. He is currently pursuing his PhD degree in electronic engineering. His research interests include macroporous silicon, photonic crystals and photovoltaic systems.

T. Trifonov author biography not currently available.

M. Garín author biography not currently available.

Á. Rodríguez (M'92-SM'08) received the B.S. degree in telecommunication engineering from the Universitat Politècnica de Catalunya, Barcelona in 1987 and the PhD degree in electronic engineering from IMEC, Belgium, in 1992. He became an Associate Professor with the Escuela Técnica Superior de Ingenieros de Telecomunicación de Barcelona in 1993. He has worked in solar cells, polysilicon TFTs, and MEMS devices. His current research topics include bio-sensors, macroporous silicon, and photonic crystals. 

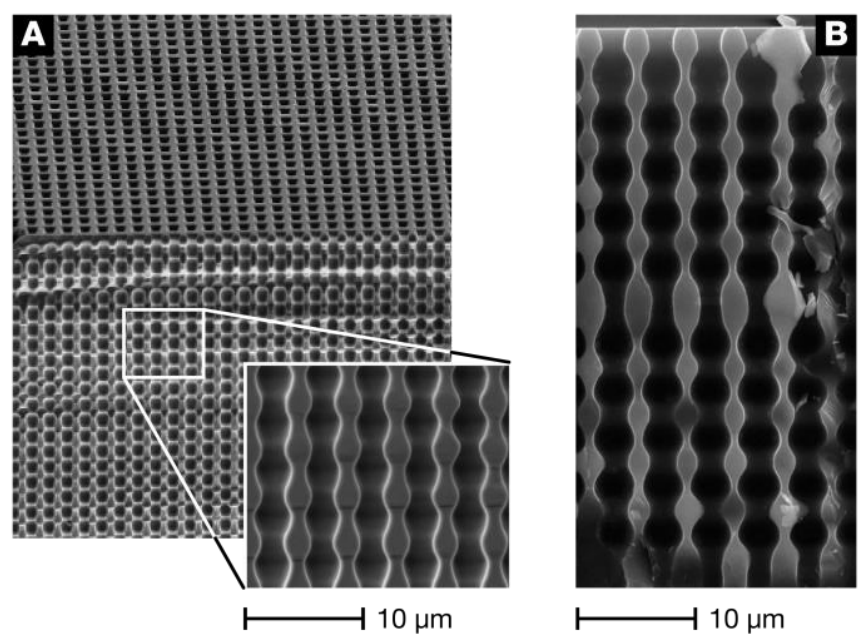

Fig. 1. Cross section view of a 3-D photonic crystal fabricated by electrochemical etching of silicon and illumination-current modulation with a sine profile. The shown sample is a $4 \mu \mathrm{m}$ pitch square lattice and identical zperiodicity. (A) shows a PC without defect. In (B) the inclusion of a defect in the PC lattice is shown. 


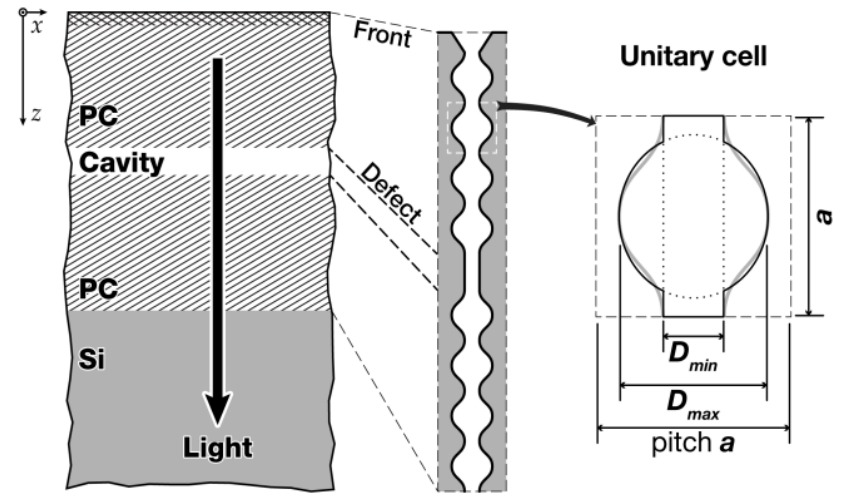

Fig. 2. Schematic representation of the structure and its mode of operation. As can be seen in the pore close-up, the initial "period" of the PC is the remaining start pit at the entrance of the pore, acting as an impedance matching element. The unitary cell is one period of the PC in the three space directions. The detailed view shows that a good match to the actual profile (grey overlay) can be achieved with an ellipsoid and a cylinder. The unitary cell detail also gives the principal design parameters. 
a)

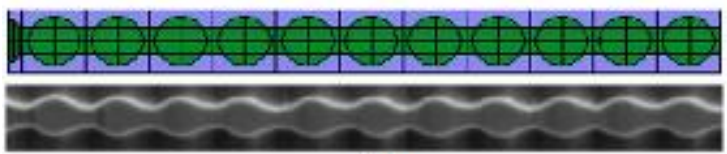

b)

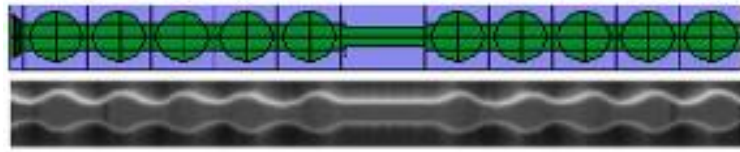

Fig. 3. Comparison of the simplified profiles used for simulation versus the real fabricated ones. In (a) a single, continuous, string of spheres approximate the ideal PC fabricated with a sinusoidal profile. In (b) a defect is introduced by removing the middle sphere and keeping a constant radius. 


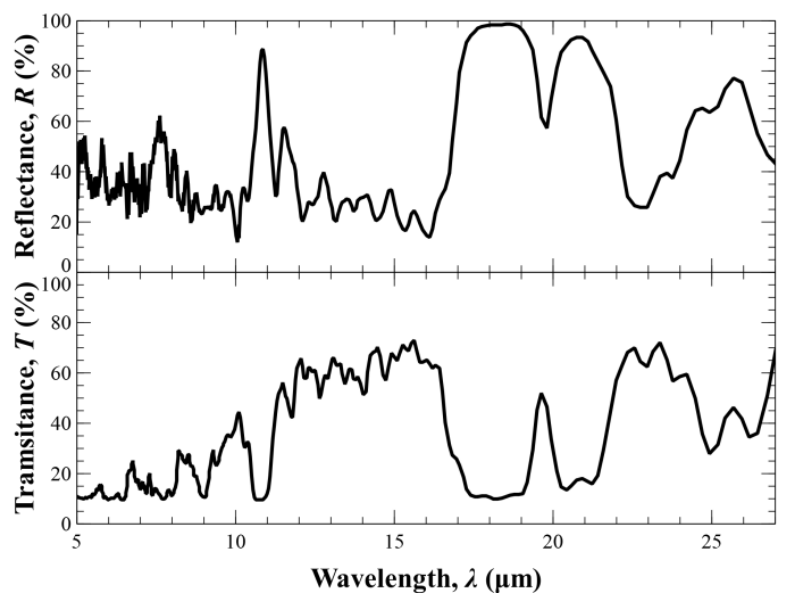

Fig. 4. Measured reflectance and transmittance spectra for a $4 \mu \mathrm{m}$ pitch sample. The PBG can be found for $\lambda$ from $15 \mu \mathrm{m}$ to $23 \mu \mathrm{m}$. The measured sample was designed with a defect which results in a clear resonance state appearing in the middle of the PBG. 


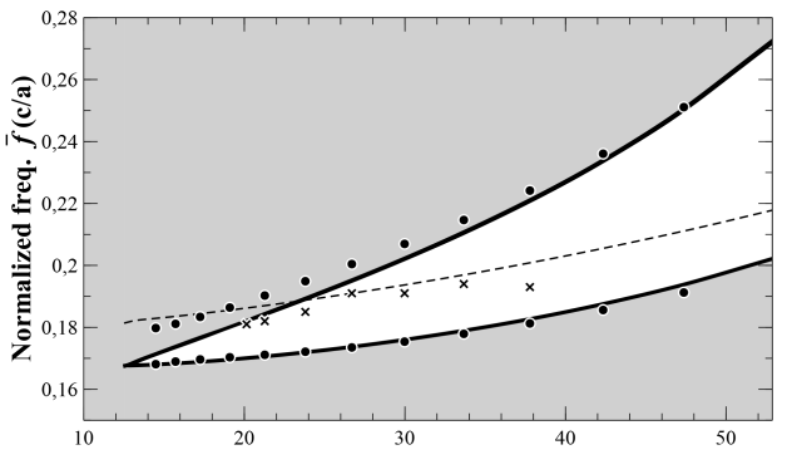

Cell Porosity $p(\%)$

Fig. 5. Photonic bandgap and defect resonant state of the simulated structure. The solid (-) and dashed (- -) lines represent the PWM simulated bands and defect resonance respectively, while the dots $(\bullet)$ and the crosses $(x)$ denote the bands and resonance respectively, obtained using the FDTD method. The PBG is obtained for several porosities of the PC structure by changing the maximum diameter if the pore. The filled area represents the propagating energies for the PC, while the clear zone is forbidden for light propagation inside the PC. When adding a defect in the structure a state appears that allows a wave in the forbidden region. 


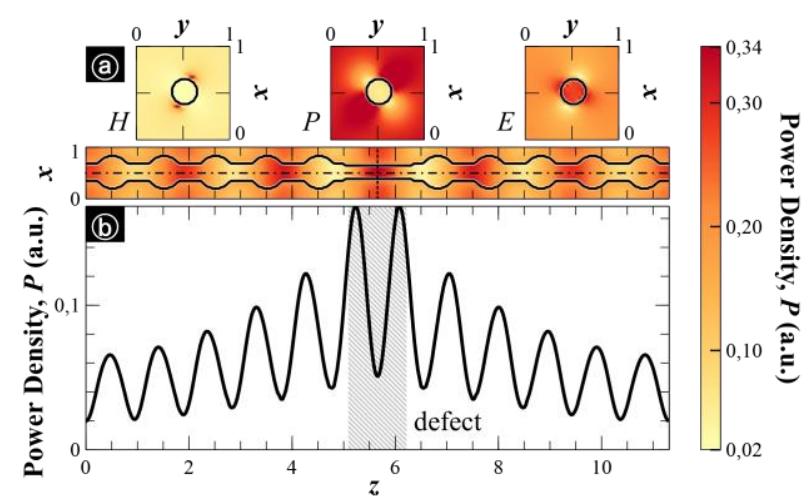

Fig. 6. EM field power density distribution inside the PC for the resonant mode. (a) shows the field distribution in $\mathrm{XZ}$ and $\mathrm{XY}$ cross-sections. The $\mathrm{XZ}$ view shows that the energy is concentrated in the defect. The $X Y$ crosssections show the $\mathrm{E}$ and $\mathrm{H}$ field distribution, and the power density at the centre of the defect. In (b) the power density along the pore axis is plotted, clearly showing that energy is stored in the defect. 


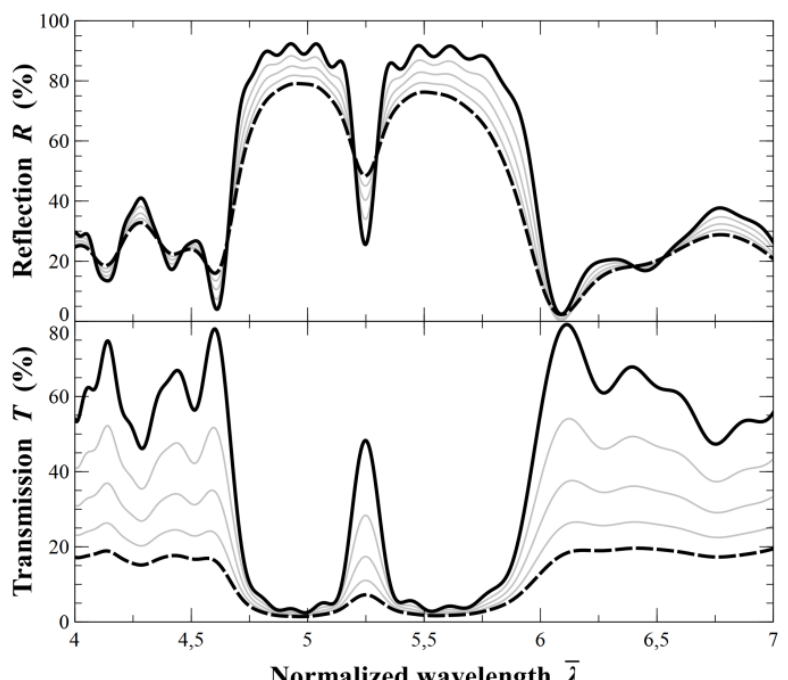

Fig. 7. FDTD simulated spectra for the PC with a defect for increasing losses. The sample porosity was $30.4 \%$. The lossless case is shown with a thick solid line while the maximum extinction factor simulated $(\mathrm{k}=0.04)$ is shown in a dashed line. Some in-between results are shown in light grey. It can be appreciated how for increasing absorption, the transmitted light is severely affected, while the reflected light is little affected. 


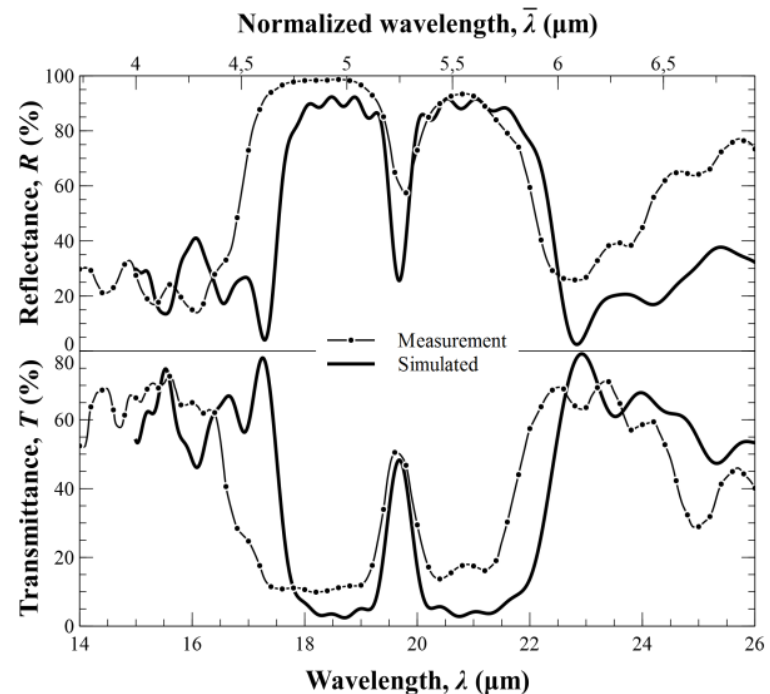

Fig. 8. Comparison of the measured results with the lossless simulated PC. The simulated spectra (solid line -) closely matches the measured spectra (dots $\bullet$ ) though there is a slight mismatch in the peak values and the obtained photonic bandgap is smaller for the simulated structure. 


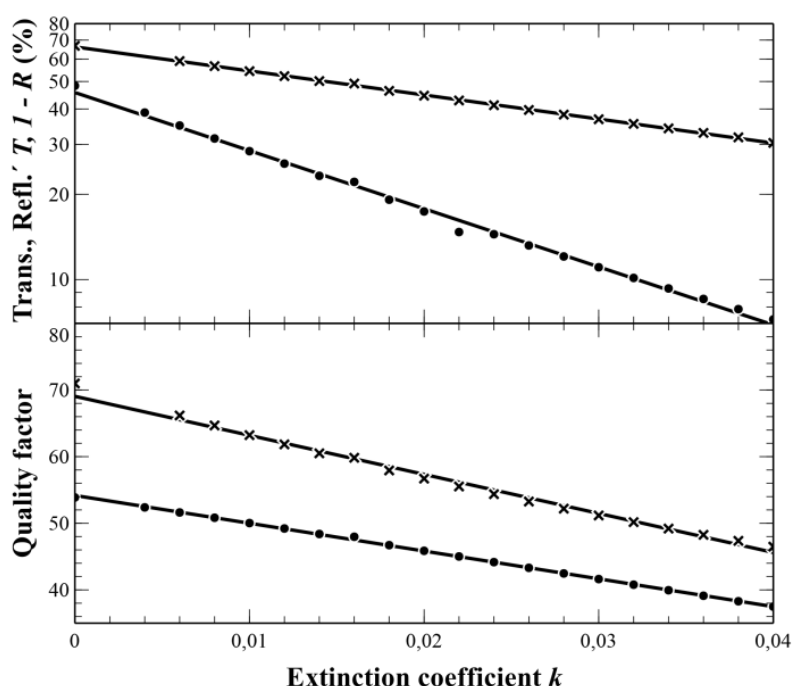

Fig. 9. Peak amplitude (top panel) and Q-factor (bottom panel) relation to the extinction factor for the reflected and transmitted light. The inverse of the reflected peaks is shown as crosses $(x)$ while the transmitted peaks are represented as dots $(\bullet)$. Sample porosity was $p=30.4 \%$. Both the reflected and transmitted light show an exponential amplitude decrease, and a linear decrease in Q-factor, with increasing extinction coefficient $\kappa$. Given the small slope of the reflected wave, it can be approximated with small error with a linear function. The sharper peak response of the reflected resonance is made clear with the higher Q-factor compared to the transmitted one. 


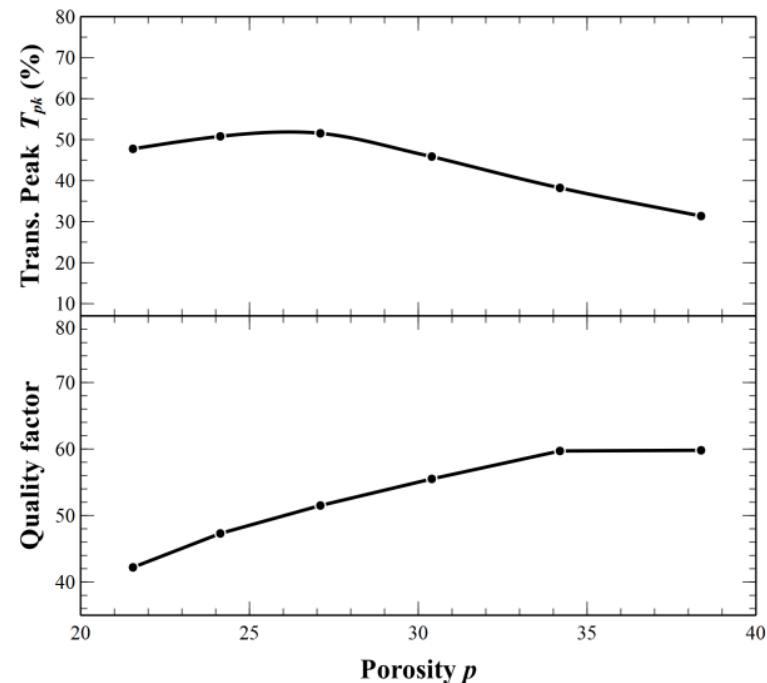

Fig. 10. Porosity dependence of the resonance peak amplitude and calculated Q-factor. The transmitted peak amplitude and its Q-factor are plotted for the lossless case vs. porosity. The Q-factor finds its maximum at about $\mathrm{p}=35 \%$ $38 \%$, but the peak maximum is obtained for a lower porosity (near $25 \%$ ). 


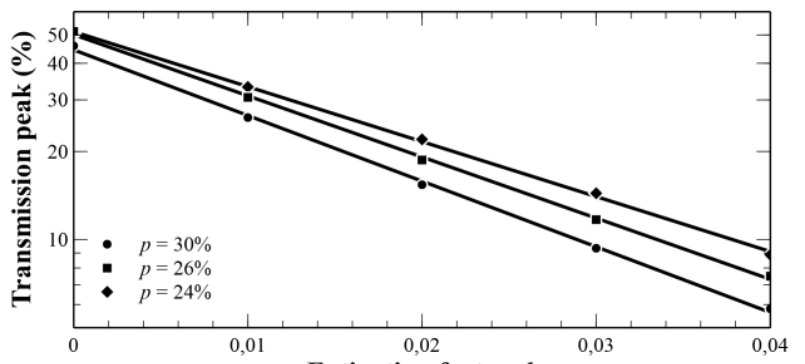

Fig. 11. Change in the peak amplitude of the transmitted light for different porosities. Shown are selected points of the transmitted light (symbols) and their exponential fit (solid line) for a sample of the studied porosities. 